\title{
Chemobrain as a Product of Growing Success in Chemotherapy - Focus on Glia as both a Victim and a Cure
}

Piotr Walczak ${ }^{1,2,3}$, Miroslaw Janowski ${ }^{1,2,+}$

\begin{abstract}
Chemotherapy-induced cognitive impairment or chemobrain is a frequent consequence of cancer treatment with many psychiatric features. Ironically, the increasing efficacy of chemotherapy leaves growing number of patients alive with chemobrain. Therefore, there is an urgent need for strategies capable of returning cancer survivors back to their pre-morbid quality of life. Molecular mechanisms of chemobrain are largely unknown. Over the last decade there was a lot of emphasis in preclinical research on inflammatory consequences of chemotherapy and oxidative stress but so far none of these approaches were translated into clinical scenario. The co-administration of chemotherapy with protective agents was evaluated preclinically but it should be introduced with caution as potential interference was not yet studied and that could blunt therapeutic efficacy. Stem cell-based regenerative medicine approach has so far been exploited very sparsely in the context of chemobrain and the focus was on indirect mechanisms or neuronal replacement in the hippocampus. However, there is evidence for widespread white matter abnormalities in patients with chemobrain. This is quite logical considering life-long proliferation and turnover of glial cells, which makes them vulnerable to chemotherapeutic agents. Feasibility of glia replacement has been established in mice with global dysmyelination where profound therapeutic effect has been observed but only in case of global cell engraftment (across the entire brain). While global glia replacement has been achieved in mice translation to clinical setting might be challenging due to much larger brain size. Therefore, a lot of attention should be directed towards the route of administration to accomplish widespread cell delivery. Techniques facilitating that broad cell distribution including intra-arterial and intrathecal methods should be considered as very compelling options. Summarizing, chemobrain is a rapidly growing medical problem and global glia replacement should be considered as worthwhile therapeutic strategy.
\end{abstract}

\section{Keywords}

Chemobrain, Chemotherapy, Cancer, Glia Progenitors, Transplantation

'The Russell H. Morgan Department of Radiology and Radiological Science, Johns Hopkins University School of Medicine, Baltimore, MD, USA

2Institute for Cell Engineering, Johns Hopkins University School of Medicine, Baltimore, MD, USA

${ }^{3}$ Department of Neurology and Neurosurgery, University of Warmia and Mazury, Olsztyn, Poland

${ }^{\dagger}$ Author for correspondences: Miroslaw Janowski, Associate Professor, The Russell H. Morgan Department of Radiology and Radiological Science, Division of MR Research Institute for Cell Engineering, Johns Hopkins University School of Medicine, CRB II 4M63, 1550 Orleans St. Baltimore, MD 21231, Tel: (443) 287 8461; Fax (443) 287 79 45; email mjanows1@jhmi.edu 


\begin{abstract}
Chemobrain
Psychological consequences of anti-cancer therapy have been raised since the 1950s [1]. Common psychiatric symptomatology among hospitalized cancer patients [2] inspired more detailed analysis of mental status in early 1980s, which revealed cognitive impairment with chemotherapy as a major associated variable $[3,4]$. Further research provided a vast body of evidence that, indeed chemotherapy is associated with short- and long-term mood alterations and cognitive deficits, characterized by disruptions in learning and memory, impaired attention, concentration, information processing speed, and executive function [5-8]. Then the term 'chemobrain' has been coined for chemotherapyinduced cognitive impairment in 2000s [9]. Among executive functions, inhibition appears relatively spared from the effects of chemotherapy, whereas impairments in shifting and updating are more common [10]. There is also a notion that self-initiated retrieval processes rather than encoding are implicated in prospective memory impairment among breast cancer survivors [11]. The recent study of patients with colorectal carcinoma treated with 5-fluorouracil with or without oxaliplatin confirmed the presence of a decline in executive function at 12 month time point comparing to patients, who had not received chemotherapy due to localized character of the disease [12].
\end{abstract}

Ironically, while increasingly effective chemotherapeutics provide a cure or satisfactory long-term control of disease more frequently, there is rapidly growing number of patients with chemobrain. This problem must be tackled to bring patients back to their premorbid quality of life. Chemobrain is most pronounced in population of patients with breast cancer with a frequency reaching $80 \%$ [13], but it also includes lung cancer $(30 \%$ of patients) [14], Central nervous system (CNS) malignancies [6], testicular cancer [15], and hematologic malignancies [16-18], and these deficits appear following treatment with various chemotherapeutic agents that have different mechanisms of action [6]. Pre-treatment cognitive screening did not reveal profound cognitive impairment [19], although some studies point to brain structure abnormalities also in cancer patients not subjected to chemotherapy [20-22]. These cognitive and psychiatric deficits are progressive and persist following the cessation of therapy [7]. Premorbid intelligence quotient
(IQ) and fatigue are also important predictors of chemobrain [23]. Even mild chemobrain-related deficits have psychiatric consequences, especially when these problems remain untreated. While immunotherapy quickly gains popularity and is not associated with cognitive deficits [24], it is only effective in $10-20 \%$ of patients, so chemotherapy is still a mainstay of cancer treatment. Given the lack of effective prevention strategies or treatments, diminished quality of life is a significant concern in survivors. As mentioned above, since patients are surviving longer following treatment, the population affected by these deficits is growing rapidly and effective treatment is urgently needed. Notably, the negative effects of chemotherapeutic drugs have been convincingly reproduced in animal models [25].

\section{- Molecular mechanisms}

The molecular mechanisms of chemobrain still need to be elucidated and that has been recognized as a priority by the National Cancer Institute which issued request for applications for broad scope neuroscience approach to chemobrain [26]. A combination of oxygen radical production and cytokine dysregulation are the main suspects for chemobrain induction [27-29]. It has been shown that aggressive, threedrug chemotherapy consisting of a combination of docetaxel, adriamycin, and cyclophosphamide led to a cytokine dysregulation and disruptions in neuroplasticity [30]. The mice subjected to chemotherapy in this study have spent less time in the target quadrant of the water maze in comparison to control mice. These findings were well aligned with decrease of manganese enhancement on magnetic resonance imaging (MRI) in the hippocampus, as well as striking elimination of dendritic spines in cortex as visualized by in vivo transcranial two-photon imaging, which indicates abnormality in neuroplasticity. These behavioral and imaging chemotherapy-induced changes highly correlated with serum and brain levels of proinflammatory cytokines: IL- 6 and TNF- $\alpha$. There was also noted decrease of anti-inflammatory cytokines IL- 4 and IL-10 in serum and brain in majority of animals subjected to investigation. These changes have been ameliorated by cotreatment with natural polyphenol-resveratrol administered orally at a daily dose $100 \mathrm{mg} / \mathrm{kg}$ [31]. The concept of cytokine dysregulation as widely occurring consequence of chemotherapy has also been a subject of a recent review [32]. In vitro experiments on $\mathrm{PC} 12$ cells using mass 
spectrometry revealed that cisplatine may affect cell membrane lipids leading to abnormal exocytotic release of neurotransmitters [33]. Alterations in gene expression dynamics found in rats following mithramycin (MTR) administration, may be responsible for observed behavioral impairment [34]. It was found that immediate molecular effects of MTR were minimal and ascribed to different unconnected trajectories, while three months later the expression of over 1000 genes was altered and they converted into specific molecular pathways. In rat a rat brain endothelial cell line (RBE4) oxaliplatin induced the disassembly of the tight junctions, the critical components of the blood brain barrier integrity, and blood components leaking into the brain may trigger brain dysfunction [35]. Carboplatin impairs dopamine release and uptake in rats as measured by fast-scan cyclic voltammetry on acute coronal brain slices post mortem [36] and whole brain preparations from zebrafish [37], which may potentially be a source of mood disturbance. Acute treatment with doxorubicin affects glutamate neurotransmission in the mouse frontal cortex and hippocampus as measured by novel glutamate-selective microelectrode arrays [38]. Notably, glutamate clearance is delayed about $50 \%$ in frontal cortex and dentate gyrus. In a case-control study in breast cancer patients mood changes were observed following everolimus, though molecular mechanisms were not investigated [39]. Fascinating observation was transgenerational induction of chemobrain in offspring from animals subjected to chemotherapy [40]. While no alteration of $\gamma \mathrm{H} 2 \mathrm{AX}$ levels suggests no DNA damage but some abnormalities in expression of proteins involved in DNA repair such as PCNA, in apoptotic pathways such as BCL2 and AKT1, and in DNA methylation including DNMT1 and MeCP2. Moreover, some alterations in expression of proteins involved in myelin production such as MBP and MYT1L were pronounced. In addition global transcriptome analysis of the whole brains displayed changes at least in 200 genes in progeny with western blot confirmation of FOXP2 and ELK1 proteins were confirmed by western blot analysis. Vascular endothelial growth factor (VEGF) signaling has been considered in the context of chemobrain; however, while plasma level of VEGF increases after anthracycline-based and taxane-based chemotherapy introduction in patients with breast cancer, it does not correlate with chemotherapy-associated cognitive impairment [41]. However, tyrosine kinase inhibitor, sunitinib malate, induces cognitive impairment in mice via dysregulation of VEGF receptor signaling. [42].

\section{- Therapeutic approaches: small molecules, through diet to stem cells}

Various therapeutic approaches have been attempted for chemobrain. A 5-fluorouracilinduced cognitive impairment in rats has been prevented with KU-32: a small molecule, C-terminal inhibitor of the molecular chaperone of heat shock protein 90 [43]. Therapeutic mechanism of $\mathrm{Ku}-32$ is likely through electrophysiological and bioenergetic neuroprotection [44]. Another small-molecule: pifithrin [45], an inhibitor of mitochondrial p53 accumulation and a popular anti-diabetic medication [46] both prevented cisplatin induced chemobrain in mice. Fluvoxamine (Flv) alleviates paclitaxel-induced $(\mathrm{Px})$ chemobrain via alleviation of endoplasmatic reticulum stress through induction of sigma-1 receptor. The purpose of this study was to investigate whether Flv could alleviate Px-induced neurotoxicity in vitro. [47]. It has been recently shown that co-administration of the antioxidant drug, 2-mercaptoethane sulfonate sodium [48,49], astaxanthin, a naturally occurring carotenoid [50], catechin, a tea polyphenol [51] as well as rutin, a glycoside abundant in citrus fruits [52] prevent doxorubicin-induced cognitive decline in animal model and the suggested mechanism is through impact on oxidative and inflammatory machineries. Of note, however that blunting oxidative stress may also potentially decrease anticancer activity [53]. It is important as it was shown before that antidepressant drugs can actually decrease cytotoxic action of temozolomide, a chemotherapeutic agent routinely used in adjuvant treatment of glioblastoma due to blood brain barrier permeability [54]. Different preventive strategies against neurotoxicity of doxorubicin and mitoxantrone were effective in vitro, which further suggests dissimilar mechanisms responsible for chemobrain. Mitoxantrone-induced toxicity was partially reverted by antioxidant, while doxorubicininduced toxicity was rather ameliorated by caspase inhibitor or protein synthesis inhibitor [55]. Female rats treated with chemotherapy mix: cyclophosphamide, methotrexate and 5-fluorouracil recovered weight faster, showed superior cognitive abilities and lower levels of depressive-like behavior when treatment with cotinine, an alkaloid found in tobacco and predominant metabolite of nicotine was 
introduced after chemotherapy [56]. It has been shown that low intensity physical exercise prevents cognitive impairment in rats by enhancing hippocampal neuroplasticity and mitochondrial function in doxorubicin-induced chemobrain [57]. Analogical study has been launched in patients to assess the value of exercise in prevention of chemobrain $[13,58]$. However, the optimism should be rather moderate regarding outcome as the wellcontrolled, randomized trial failed to show benefit of moderate to high intensity training in people with dementia [59]. This is important to notion as cognitive impairment due to chemobrain can be similar to other causes of dementia. The dietary intervention based on enrichment in long-chain, marine-derived omega-3 fatty acids and reduction of sugar intake was effective in rodent models and may accordingly reduce long-term impact of chemotherapy on cognitive decline in patients [60]. Multiple sources stated that increased activity of histone deacetylases (HDACs) play a detrimental role in chemobrain, but surprisingly sodium valproate, an inhibitor of HDACs aggravated neurotoxicity of doxorubicin in Wistar rats, thus any interventions for chemobrain needs to be introduced with caution [61].

There were two reports of stem cell-based approach to chemobrain, both in rodent models. Adriamycin-induced cerebral degenerative changes in rats were ameliorated by systemic administration of mesenchymal stem cell therapy. A reciprocal relation was recorded between the extent of regeneration and the existence of undifferentiated mesenchymal stem cells [62]. Cyclophosphamide-induced cognitive impairments in rats were resolved by intrahippocampal transplantation of human neural stem cells (ENStem-A, EMD Millipore). The satisfactory survival of engrafted cells was observed (8\%), with subsequent differentiation toward neuronal and astroglial lineages, which led to reduced neuroinflammation (significant reduction of activated microglia) and restoration of dendritic arborization [63]. None of the stem cell-based approaches to treat chemobrain has been followed by an attempt of clinical translation.

\section{- Neuroimaging}

Neuroimaging is an essential tool for effective bench-to-bed translation. White matter changes have been proposed as a hallmark of chemobrain in patients with breast cancer treated with high-dose chemotherapy with carmustine, cyclophosphamide and cisplatin with support of autologous hematopoietic progenitor cell support [64]. The long-term study in patients with breast cancer treated with cyclophosphamide, methotrexate and 5-fluorouracil has shown that such white matter abnormalities often persist decades after chemotherapy [65]. The next study including a relatively large cohort of patients (over 100 in total) subjected to various chemotherapeutic regimes revealed that small regional volumes of white matter are accompanied by gray matter shrinkage in areas involved in cognitive processing, such as grey prefrontal cortex and para-hippocampal gyrus [66]. Not surprisingly, it was then shown that there is reduction of total brain volume in patients subjected to chemotherapy, but unexpectedly no difference in volume of hippocampus was found in another study of elderly breast cancer survivors treated with 6 cycles of cyclophosphamide, methotrexate and 5-fluorouracil between 1976 and 1995 [67]. Frontal grey matter reduction correlated with executive function complaints in a study of over 20 survivors of breast cancer treated with a variety of chemotherapy regimens [68]. The white matter abnormality has been also confirmed by reduction of fractional anisotropy, and it also correlated with self-reported cognitive failure questionnaire scores [69-73]. Altered organization of global brain networks was found by resting state functional MRI (fMRI) in survivors of breast cancer treated with various regimens of chemotherapy [74]. Thus default mode network was also proposed as a biomarker of chemobrain [75]. fMRI during a working memory task revealed larger and more diffuse areas of activation in bilateral frontal and parietal regions in the twin with breast cancer treated with doxorubicin, cyclophosphamide and docetaxel [76]. Another fMRI study revealed slower reaction times in fourteen women with breast cancer subjected to chemotherapy with two regimens: cyclophosphamide, methotrexate and 5-fluorouracil or adriamycin, cyclophosphamide, and taxol/taxotere compared to controls during the recall component, even if both groups revealed similar response accuracy [77]. There was also reported reduced activity in the left lateral prefrontal cortex in 25 breast cancer survivors treated with seven various chemotherapy regimens during the card-sorting task, which required determination implicit rules governing the computer's categorization of geometric figures. It came along with increased perseverative errors and slowed processing speed 
compared with both non-chemotherapy and healthy participants [78]. No differences on the fMRI of a verbal auditory n-back task was found but high load task conditions revealed a decrease in performance at 1 month, with improvement at 1 year [79]. There was also observed altered cerebral blood flow using arterial spinal labeling MRI one month after systemic chemotherapy using several regimens in breast cancer survivors [80]. Administration of doxorubicin in naïve rats decreased ${ }^{18}$ Fluorodeoxyglucose $\left({ }^{18} \mathrm{~F}-\mathrm{FDG}\right)$ uptake only in the prefrontal cortex, which correlated well with deficits in novel object recognition while no impairment in contextual fear conditioning and these observations emphasize problems with executive functions [81]. Donepezil improved both cognitive function as well as brain glucose metabolism measured by ${ }^{18} \mathrm{~F}-\mathrm{FDG}$ positron emission tomography (PET) in rats treated with doxorubicin or cyclophosphamide [82]. No longitudinal PET study has been performed in patient with chemobrain [83], but the frontal hypometabolism was detected in pilot study by PET in ten elderly breast cancer survivors treated with standard multi-agent regimens including cyclophosphamide, methotrexate and 5-fluorouracil or an anthracycline (doxorubicin), who were at least 50 year old at diagnosis and older than 65 years at the examination as well as survived a minimum of ten years without recurrence [84]. The structural connectome approach revealed significant decrease in local efficiency, network clustering and small worldness in patients with testicular cancer recruited between June 2012 and December 2013 subjected to chemotherapy with bleomycin, etoposide and cisplatine in comparison to surgery-only group [85].

\section{Glia replacement}

\section{- Context of chemobrain}

The primary mechanism of chemotherapy is to interfere with the process of cell division and while cancer cells are intended target other dividing cells are unequivocally affected. In the brain the most studied adverse effect of chemotherapy was impairment of hippocampal neurogenesis [86,87]. Dividing cells, however, are not limited to neurons; in the adult brain, there is a continuous turnover of glial cells. Gliogenesis and myelination are dynamic and constantly modulated processes that occur in both humans and rodents, are strongly associated with neuronal activity, and are required for learning new tasks [88]. Global replacement of mouse glial cells with human counterparts improved learning and memory. This work elevates the role of glia from simply supporting function to participating in higher brain functions as well as suggests evolutionally acquired new functionality in humans [89]. Initially, these cells were shown to be therapeutic in animal models of myelin disorders, such as transverse myelitis [90] and dysmyelinations [91]. However, our preliminary experiments have shown that their therapeutic activity is far beyond re-myelination alone and seems to be primarily related to the trophic support of neurons, as latter this process occurs much faster following implantation than the production of compact myelin [92]. Moreover, the in vitro data suggests high anti-inflammatory activity of glial restricted precursors (GRPs). Glia play a prominent role in neurodegeneration, given that transplantation of GRPs derived from patients with amyotrophic lateral sclerosis recapitulate the disease in mice [93]. Similar effects were achieved with Huntington's disease, where transplantation of healthy GRPs ameliorated neurological symptoms in a mouse model [94]. The success of GRPs in animal models of myelin disorders as well as models of neurodegeneration make them a very attractive therapeutic option for psychiatric disorders, particularly where the glial component might be heavily affected, such as in chemobrain. Considering the above, we would like to encourage expanding the search for mechanisms underlaying chemobrain beyond interference with neurogenesis [95]. We hypothesize that chemotherapy may interfere with the process of gliogenesis through decreasing the pool of proliferating precursors of glia. Such disruption of gliogenesis results in insufficient support of neurons throughout the CNS, a decrease of tissue integrity, and widespread behavioral disruptions [96].

\section{- Glial progenitors}

Two different classes of glial precursors deserve attention in the context of chemobrain, first is post-developmental adult pool of tissue resident precursors as a subject to damage during chemotherapy and second, are isolated precursors meant for transplantation-based therapy to prevent or restore neurological deficits following chemotherapy. Adult brain contains tissue resident mitotically active progenitors of glia that continuously replace mature glial phenotypes. Turnover of glia throughout life has been reported for healthy post-developmental 
brain in both rodents and humans [97]. In disease states, such as local demyelination, these progenitors are responsible for the repair and remyelination [98]. Being proliferatively active, these progenitors are highly vulnerable to chemotherapeutics. Indeed, carmustine, cisplatin, and cytosine arabinoside (cytarabine), deoxyribonucleic acid (DNA) cross-linkers and an anti-metabolites, applied at clinically relevant concentrations were even more damaging to cultured progenitor cells than they are for several cancer cell lines. When administered systemically in mice, these chemotherapeutics suppressed proliferation and induced cell death of progenitor cells throughout the CNS and the effect lasted for weeks after drug administration ended [99]. More studies are needed to fully understand in what extent gliogenesis contributes towards chemobrain but there is strong evidence supporting that hypothesis and once that link is fully established, replacement of these populations will be a new interesting therapeutic approach. This approach however depends on the access to highly potent and safe transplantable glial progenitor cells. Developing brain is a rich source of various classes of glial progenitors. During development the first glial phenotype to appear is radial glia. Radial glia is recognized for their role in guiding migration of neurons and multipotency capable of differentiating towards both mature neurons and glia [100]. Later in development more cells expressing neural/ glial antigen 2 (NG2) proteoglycan (NG2 glia) were identified as progenitor pool with more restricted differentiation capacity generating both oligodendrocytes and astrocytes [101]. With their differentiation repertoire limited to glial phenotypes these cells have been called gliarestricted progenitors (GRPs). Peak gliogenesis occurs during second trimester pregnancy and robust and reproducible isolation protocols have been established to derive GRPs based on expression of surface marker A2B5 from various species including mouse [102] or human [103] fetal tissue. These multipotent glial progenitors can be collected at lower efficiency also at early postnatal stages; however, by 3 weeks after birth in rats and mice, their frequency appears to be much reduced or virtually lost [104]. The disadvantage of primary GRPs as a cell source is that they are derived from aborted human fetuses and, similar to other human-derived tissues, such as cadaveric B-islet cells, there would be a severe shortage of the cells once therapy becomes available for a larger population of patients. To address this problem protocols have been established to derive GRPs from pluripotent stem cells including induced pluripotent stem cells. However, major advantage of primary GRPs is their complete developmental commitment and very low risk of tumor formation [105].

\section{- Cell delivery}

When considering cell-based therapy for brain disorders it is important to focus on selecting appropriate strategy for cell delivery. This is particularly important when the disease is multifocal or global as in case of chemobrain where therapeutic cells need to be delivered to the entire brain and perhaps the spinal cord [95]. Stereotaxic intra-parenchymal injection has important advantage of high precision; however, transplanted cells cover only small portion of the CNS [106]. Cerebrospinal fluid (CSF) is another gateway to the brain and with large surface area of the CNS in contact with CSF it introduces opportunity for broad cell distribution. Indeed, we have shown in neonatal mice that intraventricular transplantation results in excellent, global engraftment with cells found throughout the neuraxis, including the entire length of the spinal cord [92]. Human brain however due to significantly larger size is more difficult target and thank to non-invasive MR imaging it has been shown that stem cells infused into lateral ventricle in pediatric patient are subject to sedimentation with cells accumulating within posterior portions of ventricular system [107]. Embedding the cells in injectable biomaterials is one strategy that may prevent displacement of injected cells and improve delivery precision $[108,109]$. Intravascular route is taking advantage of vast network of blood vessels and that is important advantage when global cell delivery is desired. The most straightforward and frequently utilized approach is intravenous injection and while technical ease and safety are strong advantages, cells injected intravenously are trapped by filtering organs and only very small fraction is able to reach the brain as it was shown in stroke model using Tc99m imaging [110]. To utilize intravascular delivery while avoiding trapping in filtering organs it is required that the cells are injected intraarterially. This route introduces unique opportunity to select artery feeding target structure and deploy the cells directly into that vessel potentially achieving high engraftment efficiency. Indeed, significant effort has been directed towards developing intraarterial cell delivery as safe and efficacious method. Cell labeling with iron oxide nanoparticles for real-time monitoring of 
intraarterial cell delivery facilitated visualization of cell engraftment, which proved to be highly variable [111]. Engineering of GRPs for enhanced expression of adhesion molecule VLA4 resulted in their improved homing in rat model of inflammation [112] and the same strategy was shown to enhance diapedesis of GRPs into the brain parenchyma in rats with stroke [113]. The advances in image analysis allows for a robust, single-cell, high-throughput screening for cell engineering strategies using microfluidic devices to optimize cell docking to vessel wall [114]. Intraarterial injection is associated with a risk of excessive engraftment and microembolism thus careful studies are needed to establish safety practices developing strategies for risk mitigation [115]. Intra-arterial injection was thought to provide high precision and reproducibility for cell injection; however, uncertainty about the final destination of injected cells proved to be high [111] and that was a stimulus to utilize imaging tools, particularly MRI-guidance to improve predictability and reproducibility of targeting [116].

\section{Conclusions}

Chemotherapy induces cognitive impairments, with executive function being primarily affected and these impairments are not present in patients with localized disease with no adjuvant therapy or subjected to immunotherapy. Variety of agents have been used in animal models for mitigating chemobrain, but notably most studies utilized naïve animals thus precluding testing for potential blunting of anticancer drug activity. Even though many methods to counteract chemobrain were used in animal studies, none has been effectively translated to clinical scenario. Neuroimaging revealed long-lasting white matter abnormalities in patients with chemobrain, vulnerability of glia to chemotherapy should not be a surprise because of constant turnover relying on proliferation of glial progenitors. Glial progenitors are both victims of chemotherapeutic drugs and excellent therapeutic target. Therefore, global glial replacement might be an ultimate measure to address the very compelling need for highly effective therapeutic method to eradicate chemobrain, and that strategy deserves attention and support. Global cell replacement requires effective routes for cell administration to the brain and intra-arterial and intrathecal routes seems to be particularly attractive in this aspect.

\section{Acknowledgement \\ NIH R01NS091100, NIH R01NS091110, R21NS106436, MSCRFD-3942, Johns Hopkins Dept. of Psychiatry and Behavioral Sciences Venture Discovery Award}

\section{References}

1. Sutherland AM, Orbach CE, Dyk RB, et al. The psychological impact of cancer and cancer surgery. I. Adaptation to the dry colostomy; preliminary report and summary of findings. Cancer 5(5), 857-872 (1952).

2. Craig TJ, Abeloff MD. Psychiatric symptomatology among hospitalized cancer patients. Am. J. Psychiatry 131(12), 13231327 (1974).

3. Silberfarb PM, Philibert D, Levine PM. Psychosocial aspects of neoplastic disease: II. Affective and cognitive effects of chemotherapy in cancer patients. Am. J. Psychiatry 137(5), 597-601 (1980).

4. Silberfarb PM. Chemotherapy and cognitive defects in cancer patients. Annu. Rev. Med 34(1), 35-46 (1983).

5. Dietrich J, Monje M, Wefel J, et al. Clinical patterns and biological correlates of cognitive dysfunction associated with cancer therapy. Oncologist 13(12), 1285-1295 (2008).

6. Schagen SB, Klein M, Reijneveld JC, et al. Monitoring and optimising cognitive function in cancer patients: Present knowledge and future directions. EJC. Supp/ 12(1), 29-40 (2014).

7. Dietrich J, Prust M, Kaiser J. Chemotherapy, cognitive impairment and hippocampal toxicity. Neuroscience 309(1), 224-232 (2015).

8. Bompaire F, Durand T, Léger-Hardy I, et al Chemotherapy-related cognitive impairment or $<<$ chemobrain $>>$ : concept and state of art. Geriatr. Psychol. Neuropsychiatr. Vieil 15(1), 89-98 (2017).

9. Wefel JS, Lenzi R, Theriault R, et al. 'Chemobrain' in breast carcinoma?: a prologue. Cancer 101(3), 466-475 (2004).

10. Yao C, Bernstein LJ, Rich JB. Executive functioning impairment in women treated with chemotherapy for breast cancer: a systematic review. Breast. Cancer. Res. Treat 166(1), 15-28 (2017)

11. Bedard M, Verma S, Collins B, et al. Prospective memory impairment in chemotherapy-exposed early breast cancer survivors: Preliminary evidence from a clinical test. J. Psychosoc. Oncol 34(4), 291-304 (2016).

12. Sales MVC, Suemoto CK, Apolinario D, et al. Effects of Adjuvant Chemotherapy on Cog- nitive Function of Patients With Early-stage Colorectal Cancer. Clin. Colorectal. Cancer S1533-0028(18), 30368-30372 (2018).

13. Oberste $M$, Schaffrath $N$, Schmidt K, et al. Protocol for the "Chemobrain in Motion - study" (CIM - study): a randomized placebo-controlled trial of the impact of a high-intensity interval endurance training on cancer related cognitive impairments in women with breast cancer receiving first-line chemotherapy. BMC. Cancer 18(1), 1071 (2018).

14. Simo M, Gurtubay-Antolin A, Vaquero L, et al. Performance monitoring in lung cancer patients pre- and post-chemotherapy using fine-grained electrophysiological measures. Neuroimage. Clin 18(1), 86-96 (2018).

15. Wefel JS, Vidrine DJ, Marani SK, et al. A prospective study of cognitive function in men with non-seminomatous germ cell tumors. Psychooncology 23(6), 626-633 (2014).

16. Friedman MA, Fernandez M, Wefel JS, et al. Course of cognitive decline in hematopoietic stem cell transplantation: a within-subjects design. Arch. Clin. Neuropsychol 24(7), 689-698 (2009). 
17. Jones D, Vichaya EG, Wang XS, et al. Acute cognitive impairment in patients with multiple myeloma undergoing autologous hematopoietic stem cell transplant. Cancer 119(23), 4188-4195 (2013).

18. Scherwath A, Schirmer L, Kruse M, et al. Cognitive functioning in allogeneic hematopoietic stem cell transplantation recipients and its medical correlates: a prospective multicenter study. Psychooncology 22(7), 1509-1516 (2013).

19. Nakamura ZM, Deal AM, Nyrop KA, et al. Associations of functional, psychosocial, medical, and socio-demographic factors with cognitive screening in chemotherapy naive patients with breast cancer. Psychooncology (2018).

20. Shiroishi MS, Gupta V, Bigjahan B, et al. Brain cortical structural differences between non-central nervous system cancer patients treated with and without chemotherapy compared to non-cancer controls: a cross-sectional pilot MRI study using clinically-indicated scans. Proc. SPIE. Int. Soc. Opt. Eng 10572G (2017).

21. McDonald BC, Saykin AJ. Alterations in brain structure related to breast cancer and its treatment: chemotherapy and other considerations. Brain. Imaging. Behav 7(4), 374-387 (2013).

22. McDonald BC, Conroy SK, Ahles TA, et al. Gray matter reduction associated with systemic chemotherapy for breast cancer: a prospective MRI study. Breast. Cancer. Res. Treat 123(3), 819-828 (2010).

23. Lycke M, Pottel L, Pottel $H$, et al. Predictors of baseline cancer-related cognitive impairment in cancer patients scheduled for a curative treatment. Psychooncology 26(5), 632-639 (2017)

24. Cuzzubbo S, Belin C, Chouahnia K, et al. Assessing cognitive function in patients treated with immune checkpoint inhibitors: A feasibility study. Psychooncology 27(7), 1861-1864 (2018).

25. Winocur G. Chemotherapy and cognitive impairment: An animal model approach. Can. J. Exp. Psychol 71(4), 265-273 (2017).

26. Horowitz TS, Suls J, Trevino M. A Call for a Neuroscience Approach to Cancer-Related Cognitive Impairment. Trends. Neurosci 41(8), 493-496 (2018).

27. Lacourt TE, Heijnen CJ. Mechanisms of Neurotoxic Symptoms as a Result of Breast Cancer and Its Treatment: Considerations on the Contribution of Stress, Inflammation, and Cellular Bioenergetics. Curr. Breast. Cancer. Rep 9(2), 70-81 (2017).

28. Gaman AM, Uzoni A, Popa-Wagner A, et al. The Role of Oxidative Stress in Etiopathogenesis of Chemotherapy Induced Cognitive Impairment (CICI)-"Chemobrain". Aging. Dis 7(3), 307-311 (2016).
29. Cheung YT, Ng T, Shwe M., et al. Association of proinflammatory cytokines and chemotherapy-associated cognitive impairment in breast cancer patients: a multi-centered, prospective, cohort study. Ann. Oncol 26(7), 1446-1451 (2015).

30. Shi DD, Huang YH, Lai CSW, et al. Chemotherapy-Induced Cognitive Impairment Is Associated with Cytokine Dysregulation and Disruptions in Neuroplasticity. Mol. Neurobiol 55(7), 1224-4 (2018).

31. Shi DD, Dong CM, Ho LC, et al. Resveratrol, a natural polyphenol, prevents chemotherapy-induced cognitive impairment: Involvement of cytokine modulation and neuroprotection. Neurobiol. Dis 114(1), 164173 (2018).

32. Ren X, Clair DK St, Butterfield DA. Dysregulation of cytokine mediated chemotherapy induced cognitive impairment. Pharmacol. Res 117(1), 267-273 (2017).

33. Mohammadi AS, Li X, Ewing AG. Mass Spectrometry Imaging Suggests That Cisplatin Affects Exocytotic Release by Alteration of Cell Membrane Lipids. Anal. Chem 90(14), 8509-8516 (2018).

34. Asor E, Ben-Shachar D. Gene expression dynamics following mithramycin treatment: A possible model for post-chemotherapy cognitive impairment. Clin. Exp. Pharmacol. Physiol 45(10), 1028-1037 (2018).

35. Branca JJV, Maresca M, Morucci G, et al. Oxaliplatin-induced blood brain barrier loosening: a new point of view on chemotherapy-induced neurotoxicity. Oncotarget 9(34), 23426-23438 (2018).

36. Kaplan SV, Limbocker RA, Gehringer RC, et al. Impaired Brain Dopamine and Serotonin Release and Uptake in Wistar Rats Following Treatment with Carboplatin. ACS. Chem. Neurosci 7(6) 689-699 (2016).

37. Field TM, Shin M, Stucky CS, et al. Electrochemical Measurement of Dopamine Release and Uptake in Zebrafish Following Treatment with Carboplatin. Chemphyschem 19(10), 1192-1196 (2018).

38. Thomas TC, Beitchman JA, Pomerleau F, et al., Acute treatment with doxorubicin affects glutamate neurotransmission in the mouse frontal cortex and hippocampus. Brain. Res 1672(1), 10-17 (2017).

39. Mir O, Salvador A, Dauchy S, et al. Everolimus induced mood changes in breast cancer patients: a case-control study. Invest. New. Drugs 36(3), 503-508 (2018).

40. Kovalchuk A, Inytskyy Y, Woycicki R, et al. Adverse effects of paternal chemotherapy exposure on the progeny brain: intergenerational chemobrain. Oncotarget 9(11), 10069-10082 (2018).

41. Ng T, Phey XY, Yeo HL, et al. Impact of Adjuvant Anthracycline-Based and Tax-
ane-Based Chemotherapy on Plasma VEGF Levels and Cognitive Function in Breast Cancer Patients: A Longitudinal Study. Clin. Breast. Cancer 18(5), e927-e937 (2018).

42. Abdel-Aziz AK, Mantawy EM, Said RS, et al. The tyrosine kinase inhibitor, sunitinib malate, induces cognitive impairment in vivo via dysregulating VEGFR signaling, apoptotic and autophagic machineries. Exp. Neurol 283(Pt A), 129-141 (2016).

43. Sofis MJ, Jarmolowicz DP, Kaplan SV, et al. KU32 prevents 5 -fluorouracil induced cognitive impairment. Behav. Brain. Res 329(1), 186-190 (2017).

44. Urban MJ, Pan P, Farmer KL, et al. Modulating molecular chaperones improves sensory fiber recovery and mitochondrial function in diabetic peripheral neuropathy. Exp. Neurol 235(1), 388-396 (2012).

45. Chiu GS, Maj MA, Rizvi S, et al. Pifithrin-mu Prevents Cisplatin-Induced Chemobrain by Preserving Neuronal Mitochondrial Function. Cancer. Res 77(3), 742-752 (2017).

46. Zhou W, Kavelaars A, Heijnen CJ. Metformin Prevents Cisplatin-Induced Cognitive Impairment and Brain Damage in Mice. PLoSOne 11(3), e0151890 (2016).

47. Tanimukai $\mathrm{H}$, Kudo $\mathrm{T}$. Fluvoxamine alleviates paclitaxel-induced neurotoxicity. Biochem. Biophys. Rep 4(1), 202-206 (2015).

48. Keeney JTR, Ren X, Warrier G, et al. Doxorubicin-induced elevated oxidative stress and neurochemical alterations in brain and cognitive decline: protection by MESNA and insights into mechanisms of chemotherapy-induced cognitive impairment ("chemobrain"). Oncotarget 9(54), 3032430339 (2018).

49. Aluise CD, Miriyala S, Noel T, et al. 2-Mercaptoethane sulfonate prevents doxorubicin-induced plasma protein oxidation and TNF-alpha release: implications for the reactive oxygen species-mediated mechanisms of chemobrain. Free. R adic. Biol. Med 50(11), 1630-1638 (2011).

50. El-Agamy SE, Abdel-Aziz AK, Wahdan S, et al. Astaxanthin Ameliorates Doxorubicin-Induced Cognitive Impairment (Chemobrain) in Experimental Rat Model: Impact on Oxidative, Inflammatory, and Apoptotic Machineries. Mol. Neurobiol 55(7), 57275740 (2018)

51. Cheruku SP, Ramalingayya GV, Chamallamudi MR, et al. Catechin ameliorates doxorubicin-induced neuronal cytotoxicity in in vitro and episodic memory deficit in in vivo in Wistar rats. Cytotechnology 70(1), 245-259 (2018).

52. Ramalingayya GV, Cheruku SP, Nayak PG, et al. Rutin protects against neuronal damage in vitro and ameliorates doxorubicin-induced memory deficits in vivo in Wistar rats. Drug. Des. Devel. Ther 11(1), 1011-1026 
(2017).

53. Wu TH, Karim N, Moscow JA. Distinguishing mechanisms of adverse drug reactions from mechanisms of actions of drugs. Oncotarget 9(65), 32404-32405(2018).

54. Bielecka AM, Obuchowicz E. Antidepressant drugs can modify cytotoxic action of temozolomide. Eur. J. Cancer. Care. (Engl) 26(5), e12551 (2017).

55. Almeida D, Pinho R, Correia V, et al. Mitoxantrone is More Toxic than Doxorubicin in SH-SY5Y Human Cells: A 'Chemobrain' In Vitro Study. Pharmaceuticals. (Basel) 11(2), E41 (2018).

56. larkov A, Appunn D, Echeverria V. Post-treatment with cotinine improved memory and decreased depressive-like behavior after chemotherapy in rats. Cancer. Chemother. Pharmacol 78(5), 1033-1039 (2016).

57. Park HS, Kim CJ, Kwak HB, et al. Physical exercise prevents cognitive impairment by enhancing hippocampal neuroplasticity and mitochondrial function in doxorubicin-induced chemobrain. Neuropharmacology 133(1), 451-461(2018).

58. Zimmer $\mathrm{P}$, Oberste $\mathrm{M}$, Bloch $\mathrm{W}$, et al. Impact of aerobic exercise training during chemotherapy on cancer related cognitive impairments in patients suffering from acute myeloid leukemia or myelodysplastic syndrome - Study protocol of a randomized placebo-controlled trial. Contemp. Clin. Trials 49(1), 1-5(2016)

59. Lamb SE, Sheehan B, Atherton N, et al. Dementia And Physical Activity (DAPA) trial of moderate to high intensity exercise training for people with dementia: randomised controlled trial. BMJ 361(8153), k1675 (2018).

60. Orchard TS, Gaudier-Diaz MM, Weinhold $\mathrm{KR}$, et al. Clearing the fog: a review of the effects of dietary omega- 3 fatty acids and added sugars on chemotherapy-induced cognitive deficits. Breast. Cancer. Res. Treat 161(3), 391-398 (2017).

61. Verma T, Mallik SB, Ramalingayya GV, et al. Sodium valproate enhances doxorubicin-induced cognitive dysfunction in Wistar rats. Biomed. Pharmacother 96(1), 736-741 (2017).

62. Zickri MB, Aziz DH El, Metwally HG. Histological experimental study on the effect of stem cell therapy on adriamycin induced chemobrain. Int. J. Stem. Cells 6(2), 104-112 (2013).

63. Acharya MM, Martirosian V, Chmielewski $\mathrm{NN}$, et al. Stem cell transplantation reverses chemotherapy-induced cognitive dysfunction. Cancer. Res 75(4), 676-686 (2015).

64. Brown MS, Stemmer SM, Simon JH, et al. White matter disease induced by high-dose chemotherapy: longitudinal study with MR imaging and proton spectroscopy. AJNR. Am. J. Neuroradiol 19(2), 217-221 (1998).

65. Koppelmans V, de Groot M, de Ruiter MB, et al. Global and focal white matter integrity in breast cancer survivors 20 years after adjuvant chemotherapy. Hum. Brain. Mapp 35(3), 889-899 (2014)

66. Inagaki M, Yoshikawa E, Matsuoka Y, et al. Smaller regional volumes of brain gray and white matter demonstrated in breast cancer survivors exposed to adjuvant chemotherapy. Cancer 109(1), 146-156 (2007).

67. Koppelmans V, de Ruiter MB, van der Lijn $F$, et al. Global and focal brain volume in long-term breast cancer survivors exposed to adjuvant chemotherapy. Breast. Cancer. Res. Treat 132(3), 1099-1106 (2012).

68. McDonald BC, Conroy SK, Smith DJ, et al. Frontal gray matter reduction after breast cancer chemotherapy and association with executive symptoms: a replication and extension study. Brain. Behav. Immun 30(S), 117-125 (2013)

69. Abraham J, Haut MW, Moran MT, et al. Adjuvant chemotherapy for breast cancer: effects on cerebral white matter seen in diffusion tensor imaging. Clin. Breast. Cancer 8(1), 88-91 (2008).

70. Deprez S, Amant F, Yigit R, et al. Chemotherapy-induced structural changes in cerebral white matter and its correlation with impaired cognitive functioning in breast cancer patients. Hum. Brain. Mapp 32(3), 480-493 (2011).

71. Deprez S, Amant F, Smeets A, et al. Longitudinal assessment of chemotherapy-induced structural changes in cerebral white matter and its correlation with impaired cognitive functioning. J. Clin. Onco/ 30(3), 274-281 (2012).

72. de Ruiter MB, Reneman L, Boogerd W, et al. Cerebral hyporesponsiveness and cognitive impairment 10 years after chemotherapy for breast cancer. Hum. Brain. Mapp 32(8), 1206-1219 (2011).

73. de Ruiter MB, Reneman L, Boogerd W, et al. Late effects of high-dose adjuvant chemotherapy on white and gray matter in breast cancer survivors: converging results from multimodal magnetic resonance imaging. Hum. Brain. Mapp 33(12), 2971-2983 (2012).

74. Bruno J, Hosseini SM, Kesler S. Altered resting state functional brain network topology in chemotherapy-treated breast cancer survivors. Neurobiol. Dis 48(3), 329338 (2012).

75. Kesler SR. Default mode network as a potential biomarker of chemotherapy-related brain injury. Neurobiol. Aging 35(S2), 11-19 (2014).

76. Ferguson RJ, McDonald BC, Saykin AJ, et al. Brain structure and function differences in monozygotic twins: possible effects of breast cancer chemotherapy. J. Clin. Oncol 25(25), 3866-3870 (2007).

77. Kesler SR, Bennett FC, Mahaffey ML, et al. Regional brain activation during verbal declarative memory in metastatic breast cancer. Clin. Cancer. Res 15(21), 6665-6673 (2009).

78. Kesler SR, Kent JS, O'Hara R. Prefrontal cortex and executive function impairments in primary breast cancer. Arch. Neurol 68(11), 1447-1453 (2011).

79. McDonald BC, Conroy SK, Ahles TA, et al. Alterations in brain activation during working memory processing associated with breast cancer and treatment: a prospective functional magnetic resonance imaging study. J. Clin. Oncol 30(20), 2500-2508 (2012).

80. Nudelman KN, Wang Y, McDonald BC, et al. Altered cerebral blood flow one month after systemic chemotherapy for breast cancer: a prospective study using pulsed arterial spin labeling MRI perfusion. PLoSOne 9(5), e96713 (2014).

81. Barry RL, Byun NE, Tantawy MN, et al. In vivo neuroimaging and behavioral correlates in a rat model of chemotherapy-induced cognitive dysfunction. Brain. Imaging. Behav 12(1), 87-95 (2018).

82. Lim I, oung HY, Yu AR, et al. PET Evidence of the Effect of Donepezil on Cognitive Performance in an Animal Model of Chemobrain. Biomed. Res. Int 2016(1), 6945415 (2016).

83. Li M, Caeyenberghs K. Longitudinal assessment of chemotherapy-induced changes in brain and cognitive functioning: A systematic review. Neurosci. Biobehav. Rev 92(1), 304-317 (2018).

84. Ponto LL, Menda Y, Magnotta VA, et al. Frontal hypometabolism in elderly breast cancer survivors determined by [(18)F] fluorodeoxyglucose (FDG) positron emission tomography (PET): a pilot study. Int. J. Geriatr. Psychiatry 30(6), 587-594 (2015).

85. Amidi A, Hosseini SMH, Leemans A, et al. Changes in Brain Structural Networks and Cognitive Functions in Testicular Cancer Patients Receiving Cisplatin-Based Chemotherapy. J. Natl. Cancer. Inst 109(12) (2017).

86. Seigers R, Schagen SB, Beerling W, et al. Long-lasting suppression of hippocampal cell proliferation and impaired cognitive performance by methotrexate in the rat. Behav. Brain. Res 186(2), 168-175 (2008).

87. Seigers R, Schagen SB, Coppens CM, et al. Methotrexate decreases hippocampal cell proliferation and induces memory deficits in rats. Behav. Brain. Res 201(2), 279-284 (2009).

88. Frisen J. Neurogenesis and Gliogenesis in Nervous System Plasticity and Repair. Annu. 


\section{Rev. Cell. Dev. Biol 32(1), 127-141 (2016).}

89. Han X, Chen M, Wang F, et al. Forebrain engraftment by human glial progenitor cells enhances synaptic plasticity and learning in adult mice. Cell. Stem. Cell 12(3), 342-353 (2013).

90. Walczak $P$, All AH, Rumpal N, et al. Human glial-restricted progenitors survive, proliferate, and preserve electrophysiological function in rats with focal inflammatory spinal cord demyelination. Glia 59(3), 499510 (2011).

91. Windrem MS, Schanz SJ, Guo M, et al. Neonatal chimerization with human glial progenitor cells can both remyelinate and rescue the otherwise lethally hypomyelinated shiverer mouse. Cell. Stem. Cell 2(6), 553-565 (2008)

92. Lyczek A, Arnold A1, Zhang J, et al. Transplanted human glial-restricted progenitors can rescue the survival of dysmyelinated mice independent of the production of mature, compact myelin. Exp. Neurol 291(1), 74-86 (2017).

93. Chen H, Qian K, Chen W, et al. Human-derived neural progenitors functionally replace astrocytes in adult mice. J. Clin. Invest 125(3), 1033-1042 (2015)

94. Benraiss A, Wang S, Herrlinger S, et al. Human glia can both induce and rescue as pects of disease phenotype in Huntington disease. Nat Commun 7(1), 11758 (2016).

95. Srivastava RK, Bulte JWM, Walczak $P$, et al. Migratory potential of transplanted glial progenitors as critical factor for successful translation of glia replacement therapy: The gap between mice and men. Glia 66(5), 907-919 (2018).

96. von Bernhardi R, Eugenín-von Bernhardi J, Flores B, et al. Glial Cells and Integrity of the Nervous System. Adv. Exp. Med. Biol 949(1), 1-24 (2016).

97. Yeung MS, Zdunek S1, Bergmann O, et al. Dynamics of oligodendrocyte generation and myelination in the human brain. Cell 159(4), 766-774 (2014).

98. Prineas JW, Barnard RO, Kwon EE, et al.
Multiple sclerosis: remyelination of nascent lesions. Ann. Neurol 33(2), 137-151 (1993).

99. Dietrich J, Han R, Yang Y, et al. CNS progenitor cells and oligodendrocytes are targets of chemotherapeutic agents in vitro and in vivo. J. Biol 5(7), 22 (2006)

100.Gotz M, Huttner WB. The cell biology of neurogenesis. Nat. Rev. Mol. Cell. Biol 6(10), 777-88 (2005).

101.Gregori N, Pröschel C, Noble M, et al. The tripotential glial-restricted precursor (GRP) cell and glial development in the spinal cord: generation of bipotential oligodendrocyte-type-2 astrocyte progenitor cells and dorsal-ventral differences in GRP cell function. J. Neurosci 22(1), 248-256 (2002).

102.Phillips AW, Falahati S, DeSilva R, et al. Derivation of glial restricted precursors from E13 mice. J. Vis. Exp (64) 3462 (2012).

103.Sandrock RW, Wheatley W, Levinthal C, et al. Isolation, characterization and preclinical development of human glial-restricted progenitor cells for treatment of neurological disorders. Regen. Med 5(3), 381-394 (2010).

104.Laywell ED, Rakic P, Kukekov VG, et al. Identification of a multipotent astrocytic stem cell in the immature and adult mouse brain. Proc. Natl. Acad. Sci. USA 97(25), 13883-13888 (2000).

105.Windrem MS, Nunes MC, Rashbaum WK et al. Fetal and adult human oligodendrocyte progenitor cell isolates myelinate the congenitally dysmyelinated brain. Nat. Med 10(1), 93-97 (2004).

106.Janowski M, Engels C, Gorelik M, et al. Survival of neural progenitors allografted into the CNS of immunocompetent recipients is highly dependent on transplantation site. Cell. Transplant 23(2), 253-262 (2014).

107.Janowski M, Walczak P, Kropiwnicki T, et al. Long-term MRI cell tracking after intraventricular delivery in a patient with global cerebral ischemia and prospects for magnetic navigation of stem cells within the CSF. PLoSOne 9(2), e97631 (2014).

108.Malysz-Cymborska I, Golubczyk D,
Kalkowski L, et al. MRI-guided intrathecal transplantation of hydrogel-embedded glial progenitors in large animals. Sci. Rep 8(1), 16490 (2018)

109. Oliveira JM, Carvalho L, Silva-Correia J, et al. Hydrogel-based scaffolds to support intrathecal stem cell transplantation as a gateway to the spinal cord: clinical needs, biomaterials, and imaging technologies. NPJ. Regen. Med 3: p. 8 (2018).

110.Detante O, Moisan A, Dimastromatteo J, et al. Intravenous administration of 99mTc-HMPAO-labeled human mesenchymal stem cells after stroke: in vivo imaging and biodistribution. Cell. Transplant 18(12), 1369-1379 (2009).

111. Walczak P, Zhang J, Gilad AA, et al. Dual-modality monitoring of targeted intraarterial delivery of mesenchymal stem cells after transient ischemia. Stroke 39(5), 1569-1574 (2008)

112.Gorelik M, Orukari I, Wang J, et al. Use of MR cell tracking to evaluate targeting of glial precursor cells to inflammatory tissue by exploiting the very late antigen-4 docking receptor. Radiology 265(1), 175185 (2012).

113.Jablonska A, Shea DJ, Cao S, et al. Overexpression of VLA-4 in glial-restricted precursors enhances their endothelial docking and induces diapedesis in a mouse stroke model. J. Cereb. Blood. Flow. Metab 38(5), 835-846 (2018).

114.Andrzejewska A, Nowakowski A, Grygorowicz $T$, et al. Single-cell, high-throughput analysis of cell docking to vessel wall. J. Cereb. Blood. Flow. Metab 271678(X1), 8805238 (2018)

115.Janowski M, Lyczek A, Engels C, et al. Cell size and velocity of injection are major determinants of the safety of intracarotid stem cell transplantation. J. Cereb. Blood. Flow. Metab 33(6), 921-927 (2013).

116.Walczak P, Wojtkiewicz J, Nowakowski A, et al. Real-time MRI for precise and predictable intra-arterial stem cell delivery to the central nervous system. J. Cereb. Blood. Flow. Metab 37(7), 2346-2358 (2017). 